\title{
Comparative in-vitro activity of fleroxacin and other 6-fluoroquinolones against mycobacteria
}

\author{
Max Salfinger ${ }^{a *}$, Peter Hohl $^{b}$ and Felicitas M. Kafader ${ }^{a}$ \\ ${ }^{a}$ Department of Medical Microbiology, University of Zürich; ${ }^{b}$ Pharma Research \\ Department, F. Hoffmann-La Roche, Switzerland
}

\begin{abstract}
The susceptibility of 11 clinical isolates of Mycobacterium tuberculosis, 3 M. kansasii, $3 \mathrm{M}$. xenopi, $2 \mathrm{M}$. scrofulaceum, $2 \mathrm{M}$. marinum, $2 \mathrm{M}$. malmoense to fleroxacin, ciprofloxacin, norfloxacin, rifampicin, isoniazid, ethambutol, and streptomycin was determined by the standard proportion method (Middlebrook 7H10 agar). All M. tuberculosis, M. kansasii, M. xenopi, M. scrofulaceum, M. marinum, and $M$, malmoense isolates including those resistant to conventional antimycobacterials were inhibited by $0.5 \mathrm{mg} / 1$ of fleroxacin and ciprofloxacin, the lowest tested concentration. Fleroxacin and ciprofloxacin along with ofloxacin, pefloxacin, ansamycin, clofazimine and cycloserine were also tested against 14 isolates of the $M$. avium complex. Nine of 14 strains $(64 \%)$ of the $M$. avium complex were found susceptible to $4 \mathrm{mg} / \mathrm{l}$ of fleroxacin and a similar percentage to the other quinolones. On the basis of its in-vitro potency and its favourable pharmacokinetic properties fleroxacin appears to be sufficiently active to warrant further experimental trials against difficult to treat mycobacteria.
\end{abstract}

\section{Introduction}

Fleroxacin (Ro 23-6240, AM-833), the 6,8 difluoro-1-(2-fluoroethyl)-1,4-dihydro7-(4-methyl-1-piperazinyl)-4-oxo-quinoline-3-carboxylic acid has been shown to be highly active against a broad spectrum of Gram-positive and Gram-negative aerobic micro-organisms with the exception of many streptococci and anaerobes (Chin, Brittain \& Neu, 1986). However, the antimycobacterial activity of this newly discovered 6-fluoroquinolone has not been as well characterized as other sectors of its antibacterial spectrum.

The purpose of the present study was to determine the in-vitro activity of fleroxacin in comparison with ciprofloxacin and norfloxacin against selected strains of susceptible and multiply resistant Mycobacterium tuberculosis, and against unselected isolates of the $M$. avium complex and of a further six clinically important species of mycobacteria other than tubercle bacilli (MOTT). Concurrently, the susceptibility of the strains belonging to the $M$. avium complex was also determined to ofloxacin, pefloxacin, ansamycin, clofazimine and D-cycloserine, and that of all the other mycobacteria mentioned to the first-line antituberculous compounds.

\footnotetext{
*Present address: Max Salfinger, M.D., Webb-Waring Lung Institute, 4200 East 9th Avenue, B-122 Denver, CO 80262, USA.

Corresponding author: Peter Hohl, M.D., Pharma Research Department, PF/ID, F. Hoffmann-La Roche, CH-4002 Basle, Switzerland.
} 


\section{Materials and methods}

\section{Antimicrobial drugs}

The following compounds were provided as standard laboratory powders by the listed suppliers: fleroxacin [Ro 23-6240; AM-833 (F. Hoffmann-La Roche \& Co. Ltd., Basle, Switzerland)]; ansamycin [(LM-427, a spiropiperidyl rifamycin); Farmitalia Carlo Erba, Milan, Italy]; ciprofloxacin (Bayer AG, Wuppertal West Germany); clofazimine (Ciba-Geigy, Basle, Switzerland); and D-cycloserine (F. Hoffmann-La Roche \& Co. Ltd., Basle, Switzerland). Pefloxacin (Laboratoires Roger Bellon, Neuilly-s.-Seine, France), ofloxacin (Hoechst AG, Frankfurt a. Main, West Germany), norfloxacin (Merck, Sharp \& Dohme, West Point, PA, USA) as well as rifampicin, streptomycin, ethambutol, and D-cycloserine were obtained from commercial sources.

\section{Isolates}

Thirty seven isolates $(11 \mathrm{M}$. tuberculosis, $14 \mathrm{M}$. avium complex, $3 \mathrm{M}$. kansasii, 3 $M$. xenopi, $2 M$. scrofulaceum, $2 \mathrm{M}$. malmoense, $2 \mathrm{M}$. marinum) were studied. All organisms were drawn from the stock culture collection of recent clinical isolates of the Department of Medical Microbiology of the University of Zürich except for those provided by Dr P. A. Jenkins, PHLS Mycobacterium Reference Unit, University Hospital of Wales, Cardiff, UK (3 strains of $M$. avium complex) and $\mathrm{Dr} \mathrm{K} . \mathrm{H}$. Schroeder, Forschungsinstitut Borstel, West Germany ( 2 M. avium complex). The strains were identified by standard methods (Sommers \& Good, 1985) and kept on Lowenstein-Jensen slants up to the time of use. Of the 11 strains of $M$. tuberculosis, 6 were susceptible and 5 resistant to both isoniazid and rifampicin. Of the 5 resistant isolates, 3 were also resistant to ethambutol and 2 to streptomycin. $M$. tuberculosis $\mathrm{H} 37 \mathrm{Rv}$ was used as the control strain throughout the trial.

\section{Susceptibility testing}

Susceptibility testing was performed by the proportion method (Vestal, 1981; Sommers \& Good, 1985; McClatchy, 1986). In brief, fleroxacin, ciprofloxacin, ofloxacin and norfloxacin were each solubilized in $0.06 \mathrm{ml}$ of $1 \mathrm{~N} \mathrm{NaOH}$ and $1 \mathrm{ml}$ of methanol and, along with pefloxacin and D-cycloserine, diluted in sterile water to the desired final concentration. In contrast, ansamycin was dissolved in dimethylformamide and diluted in Sorensen buffer ( $\mathrm{pH} \mathrm{6.8),} \mathrm{and} \mathrm{clofazimine} \mathrm{in}$ absolute ethanol, respectively. Compounds were adjusted for solvent, water and impurity content.

Fresh solutions of the antibacterial agents were prepared on the day of the experiment, filter sterilized (0.22 $\mu \mathrm{m}$ Millipore) and, in the case of fleroxacin, stored in the dark up to the time of use. Appropriately concentrated aliquots $(0.25 \mathrm{ml})$ of each drug were placed in three of four quadrants of each plate (the remaining quadrant being the antimicrobial-free control sector) and mixed with cooled molten $\left(52^{\circ} \mathrm{C}\right)$ agar [Middlebrook 7H10 agar (Difco Laboratories, Detroit, MI, USA) containing 10\% (v/v) Middlebrook OADC supplement (Difco)]. The final volume was $5 \mathrm{ml}$ per quadrant ( $85 \mathrm{~mm}$ Petri dish), and final concentrations of antimicrobials tested were $0.5,2$ and $8 \mathrm{mg} / 1$ for the 6-fluoroquinolones against $M$. tuberculosis and most MOTT, and 2,4 , and $8 \mathrm{mg} / 1$ against the $M$. avium complex. Other final concentrations 
employed were $2 \mathrm{mg} / 1$ for ansamycin and streptomycin, $1 \mathrm{mg} / \mathrm{l}$ for clofazimine, rifampicin and isoniazid, $10 \mathrm{mg} / 1$ for ethambutol, and 30 and $60 \mathrm{mg} / 1$ for D-cycloserine.

In preparation for inoculation mycobacteria were grown in Middlebrook $7 \mathrm{H} 9$ broth supplemented with Middlebrook OADC enrichment (Difco) at $37^{\circ} \mathrm{C}$ for five days, days, then diluted with saline and matched to a McFarland No. 1 standard. The resulting suspension was carefully vortexed for optimal homogenization prior to two consecutive $1: 20$ dilutions. Aliquots ( $150 \mu \mathrm{l}$ per quadrant) from each of these three successive dilutions were used to seed the entire series of test plates set up for a particular strain including growth controls. Likewise, aliquots from the respective series of bacterial suspensions were used to demonstrate on other control plates that the solvents at the concentrations used in the trial were not inhibitory to the mycobacteria tested.

Cultures were incubated at $37^{\circ} \mathrm{C}$ in air with 5 to $10 \% \mathrm{CO}_{2}$ (except for $M$. marinum kept at $30^{\circ} \mathrm{C}$ in room air). Plates were read after three weeks' incubation with a stereomicroscope. Drug activity was assessed by dividing the number of colony forming units on quadrants with antimycobacterial by that of the respective drug-free control quadrant on the same plate growing preferentially 200-700 colonies.

Inhibitory activity of the test compounds was defined as the lowest concentration of antimycobacterial that reduced the colony count to less than $1 \%$ of that observed on the antimicrobial-free quadrant. Conversely, mycobacterial growth of $1 \%$ or greater on the plate with the highest listed or with the only stated concentration of antimycobacterial compound was interpreted as full resistance, and growth at the lower of two stated clinically achievable concentrations as partial resistance to a given compound.

Stability of fleroxacin in Middlebrook 7 H9 broth (Difco, Lot. No. 673876) and $0.2 \%$ glycerol (Merck B 42522) was determined by high-performance liquid chromatography with a fluorimetric detector (SMF, Kontron) using $0 \cdot 15 \%$ sodium dodecyl sulphate in acetonitrile-water-buffer $\mathrm{pH} 2$ (Titrisol, Merck) as mobile phase for both the precolumn (Pellicular $\mathrm{ODS}^{\circledR}, 40 \mu \mathrm{m}$, Whatman) and the column (Novapack ${ }^{\circledast}, 4 \mu \mathrm{m} ; 150 \times 3.9 \mathrm{~mm}$ ID, Waters). Aliquots of broth were removed from incubation at $37^{\circ} \mathrm{C}$ in the dark (fleroxacin being susceptible to degradation by light after exposure over several days) and sampled on days 0,7 , and 28 . The coefficient of variation of our assay system was less than $5 \%$.

\section{Results}

\section{Stability}

Experiments revealed that the potency of fleroxacin $(10 \mathrm{mg} / \mathrm{l})$ was fully maintained in the dark at $4{ }^{\circ} \mathrm{C}$ in $0.9 \%$ sodium chloride and in $4 \%$ reconstituted human albumin over a four week storage period (data not shown). In experiments with Middlebrook $7 \mathrm{H} 9$ broth storage at $37^{\circ} \mathrm{C}$ with or without $0.025 \mathrm{mg} \%$ malachite green, the potency of fleroxacin degraded by $23 \%$ without and by $25 \%$ with malachite green over a 28 day period (data not shown).

\section{Antimycobacterial activity}

Results on the activity of fleroxacin and six comparative compounds against M. tuberculosis (with some strains being resistant to both rifampicin and isoniazid) 


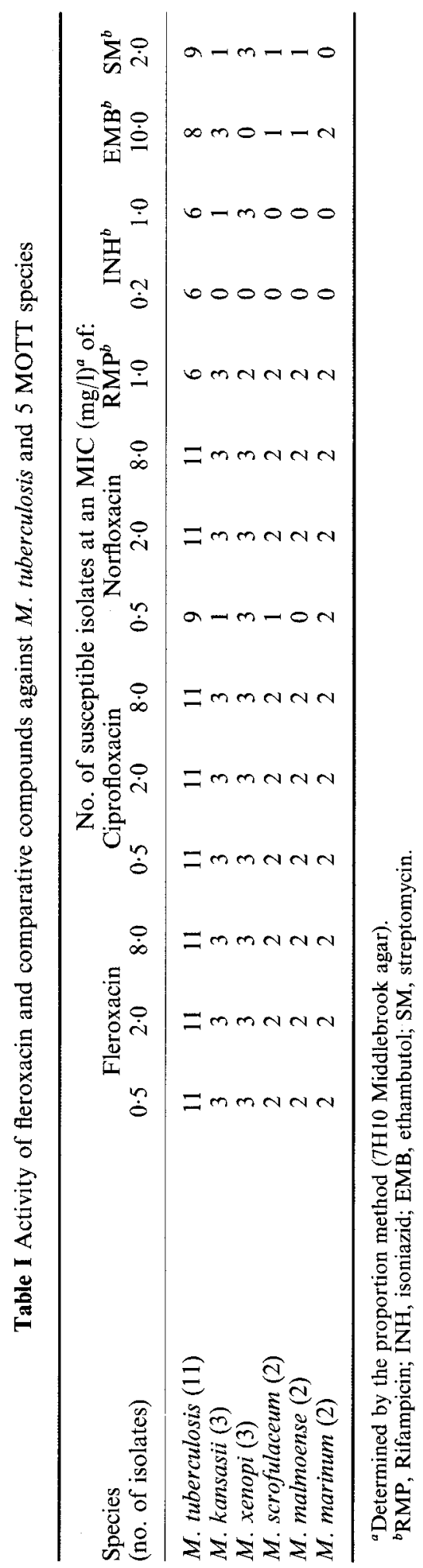


and five MOTT species other than $M$. avium complex are summarized (Table I). All 23 strains, including the control strain H37Rv, were found to be susceptible to fleroxacin and ciprofloxacin at $0.5 \mathrm{mg} / 1$, the lowest concentration tested. In contrast, only 16 $(70 \%)$ of 23 tested isolates were susceptible to norfloxacin at $0.5 \mathrm{mg} / 1$ even though $100 \%$ of strains were inhibited by a concentration of $2 \mathrm{mg} / \mathrm{l}$. Thus, norfloxacin was less potent $(\mathrm{W} / \mathrm{W})$ than the other quinolones against M. tuberculosis, M. kansasii, $M$. scrofulaceum and $M$. malmoense. As expected, rifampicin and isoniazid were inactive at the tested concentrations against multi-resistant $M$. tuberculosis even though the latter were quinolone-susceptible. Isoniazid, but not rifampicin, was inactive also against most MOTT strains examined.

Some of the 14 strains of the $M$. avium complex were partially resistant to the 6-fluoroquinolones. The activity of fleroxacin as measured was similar to that of ciprofloxacin, norfloxacin and ofloxacin but slightly weaker than that of pefloxacin (Table II). At concentrations of $4 \mathrm{mg} / 1$ and $8 \mathrm{mg} / 164 \%$ and $79 \%$ of tested isolates were susceptible to fleroxacin and ciprofloxacin as against $71 \%$ and $93 \%$ to pefloxacin. All isolates resistant to $8 \mathrm{mg} / 1$ of fleroxacin were also resistant to $8 \mathrm{mg} / \mathrm{l}$ of ciprofloxacin, norfloxacin, and ofloxacin. One strain was even uniformly resistant to all five quinolones at the highest tested concentration of $8 \mathrm{mg} / \mathrm{l}$.

Table II. Activity of fleroxacin and seven comparative compounds against $M$. avium complex (14 strains)

\begin{tabular}{lccc}
\hline Drug & $\begin{array}{c}\text { Concentration } \\
(\mathrm{mg} / \mathrm{l})\end{array}$ & $\begin{array}{c}\text { Number } \\
\text { susceptible }\end{array}$ & $\begin{array}{c}\text { Strains } \\
\%\end{array}$ \\
\hline Fleroxacin & 2 & 6 & 43 \\
& 4 & 9 & 64 \\
Ciprofloxacin & 8 & 11 & 79 \\
& 2 & 7 & 50 \\
Norfloxacin & 4 & 9 & 64 \\
& 8 & 11 & 79 \\
Ofloxacin & 2 & 4 & 29 \\
& 4 & 11 & 50 \\
Pefloxacin & 8 & 4 & 79 \\
& 2 & 8 & 29 \\
Ansamycin & 4 & 11 & 57 \\
Clofazimine & 8 & 6 & 79 \\
Cycloserine & 2 & 10 & 43 \\
& 4 & 13 & 71 \\
& 2 & 14 & 93 \\
\hline
\end{tabular}

${ }^{a}$ Susceptible, determined by the proportion method (Canetti) on Middlebrook $7 \mathrm{H} 10$ agar. 
Interestingly, the quinolone resistant strains of the $M$. avium complex were mainly susceptible in-vitro to the three non-quinolone drugs, and conversely, the two clofazimine resistant (MIC $>1 \mathrm{mg} / \mathrm{l})$ isolates as well as the four D-cycloserine resistant (MIC $>30 \mathrm{mg} / \mathrm{l}$ ) strains of the $M$. avium complex were inhibited by all quinolones tested and by ansamycin. D-cycloserine was inhibitory to ten strains of the $M$. avium complex at a concentration of $30 \mathrm{mg} / 1$ and to all 14 strains at a concentration of $60 \mathrm{mg} / 1$ (peak serum level of D-cycloserine $20-50 \mathrm{mg} / 1$ ). Similarly, $2 \mathrm{mg} / \mathrm{l}$ of ansamycin, a congener of rifamycin-S, were inhibitory in vitro to all $M$. avium complex isolates included in the trial.

\section{Discussion}

There still is an urgent need for new drugs to improve the prognosis of patients suffering from mycobacterial diseases due to drug-resistant $M$. tuberculosis or nontuberculous mycobacteria. Cure rates of infections due to multiresistant M. tuberculosis (Costello, Caras \& Snider, 1980; Bass, 1986; Jones et al., 1987) and drug resistant MOTT have remained unsatisfactory despite combination therapy (Greene et al., 1982; Masur et al., 1987). Because of the recently discovered high incidence of mycobacterial infections in patients with AIDS (Goldman, 1987), there is an increasing demand for new first-line antimycobacterial agents that cure even the most severe forms of opportunistic $M$. avium complex infections in patients afflicted with the HIV-induced immunodeficiency (Horsburgh et al., 1986), and of other mycobacterial diseases that are refractory to currently used antimycobacterial drugs as well.

The present study describes the in-vitro activity of the new 6-fluoroquinolone fleroxacin in comparison to that of ciprofloxacin, norfloxacin and other drugs against some very difficult to treat mycobacteria. In these susceptibility determinations, which were limited to antibacterial concentrations near or at the (proposed) susceptibility breakpoints of the various quinolones under study (Wise et al., 1986, 1987), fleroxacin showed virtually the same degree of in-vitro activity $(\mathrm{w} / \mathrm{w})$ as ciprofloxacin against multiply resistant $M$. tuberculosis and six species of MOTT other than $M$. avium complex.

Fenlon \& Cynamon (1986) found that the $\mathrm{MIC}_{90}$ of ciprofloxacin and norfloxacin were 0.5 and $4 \mathrm{mg} / 1$ for $M$. tuberculosis isolates, respectively. Thus, there was good agreement between their study and our results. Using 7H11 Middlebrook agar, Gay, DeYoung \& Roberts (1984) and Berlin, Young \& Bruckner (1987) reported somewhat higher MICs for ciprofloxacin $\left(\mathrm{MIC}_{90}\right.$ of $\left.1 \mathrm{mg} / \mathrm{l}\right)$ and norfloxacin and ciprofloxacin $\left(\mathrm{MIC}_{90}\right.$ of 8 and $2 \cdot 0 \mathrm{mg} / \mathrm{l}$, respectively) against $M$. tuberculosis. Collins \& Uttley (1985) reporting on the activities of ciprofloxacin against $M$. tuberculosis and its geographical variants, M.xenopi, M. marinum, M.kansasii, and M. aviumintracellulare-scrofulaceum, published that 164 out of 207 isolates (all except $M$. aviumintracellulare-scrofulaceum) had ciprofloxacin MICs of $1.56 \mathrm{mg} / \mathrm{l}$ or less in LowensteinJensen medium. These values were somewhat higher than those observed by us. Methodological differences between the laboratories and varying susceptibility in different strains may account for the three-fold disparity in MICs. In the present study, two strains of $M$. malmoense were found susceptible to $0.5 \mathrm{mg} / 1$ of fleroxacin and ciprofloxacin, whereas others (Davies, Sparham \& Spencer, 1987) have reported lower activity (MIC of $5 \mathrm{mg} / \mathrm{l}$ or greater) for ciprofloxacin against these organisms. 
The results of fleroxacin and ciprofloxacin against 14 isolates of $M$. avium complex agreed well with those of Gay et al. (1984) who reported on the activity of ciprofloxacin $\left(\mathrm{MIC}_{90}\right.$ at $\left.16 \mathrm{mg} / \mathrm{l}\right)$ and of norfloxacin $\left(\mathrm{MIC}_{90}>16 \mathrm{mg} / \mathrm{l}\right)$ against these organisms. Fenlon and Cynamon (1986) found lower MICs to ciprofloxacin $\left(\mathrm{MIC}_{90}\right.$ $2 \mathrm{mg} / \mathrm{l})$ and to ofloxacin $\left(\mathrm{MIC}_{90} 8 \mathrm{mg} / \mathrm{l}\right)$ for $M$. intracellulare than our study. The observation of Tsukamura (1983) might explain the discrepancy. This author found that $M$. intracellulare was inhibited by lower concentrations of ofloxacin than $M$. avium. The reason for the divergence in activity of ofloxacin against the two species, $M$. avium or $M$. intracellulare, could account for correspondingly diverging activity of other quinolones. Yet irrespective of whether the choice of strains could have influenced the results in favour of the compounds, ofloxacin did not quite match the activity (w/w) of ciprofloxacin in Fenlon \& Cynamon's study (1986) nor that of ciprofloxacin and fleroxacin in the present study. Pefloxacin was also active in the present trial against $M$. avium complex. We are unaware that this finding has been reported to date in the literature. Whether the pefloxacin activity would be confirmed in the clinical situation where the drug undergoes metabolism (Wise et al., 1987) remains to be proven.

Heifets \& Iseman (1985) tested 523 isolates of $M$. avium complex and found that $95 \%$ were susceptible to $2 \mathrm{mg} / 1$ of ansamycin. The $\mathrm{MIC}_{90}$ for $20 \mathrm{M}$. intracellulare isolates was $1 \mathrm{mg} / \mathrm{l}$ of ansamycin in the series reported by Cynamon (1985) and $2 \mathrm{mg} / \mathrm{l}$ in that published by Kiehn et al. (1985). In addition, the 35 isolates of Kiehn et al. (1985) were susceptible to cycloserine ( 30 and $60 \mathrm{mg} / 1)$ and clofazimine $(1 \mathrm{mg} / \mathrm{l})$. Our data agreed generally with these results but unlike these authors we encountered two strains resistant to clofazimine $(1 \mathrm{mg} / 1)$ and four strains resistant to D-cycloserine $(30 \mathrm{mg} / \mathrm{l})$. The proven clinical results with ansamycin and clofazimine singly or in combination against $M$. avium infections (Whimbey, Kiehn \& Armstrong, 1986) and the promising in-vitro activity of fleroxacin and the other new 6-fluoroquinolones raise the hope that with the advent of these new compounds the future of patients afflicted with $M$. avium complex infection could be less troublesome than at present.

Fleroxacin with its bioavailability of $100 \%$ after oral absorption (tablet), a low degree of metabolism and a long plasma elimination half-life of 9-10 h (Weidekamm, et al., 1987) is known to combine not only a wide spectrum of activity similar to that of its congeners but also promising pharmacokinetics that distinguish it from the earlier quinolones such as the ones used comparatively in this trial (Wise et al., 1986, 1987). Its rapid and uniform penetration into blister fluid in volunteers with peak levels in excess of $3 \mathrm{mg} / \mathrm{l}$ following a standard $400 \mathrm{mg}$ oral dose suggests that some of the pathogenic mycobacteria tested might respond to the compound and that the compound is worth further experimental investigations against these organisms including killing tests (Yajko, Nassos \& Hadley, 1987).

\section{Acknowledgements}

The work presented herein was supported in part by a grant from F. Hoffmann-La Roche \& Co. Ltd., Basle, and presented at the 46th Annual Meeting of the American Thoracic Society, Kansas City, Missouri, May 11-14, 1986, poster presentation 287.

We thank F. Hoffmann-La Roche \& Co. Ltd., Basle, Switzerland for supplying fleroxacin. Ciba-Geigy Ltd., Basle, Switzerland kindly provided clofazimine, Bayer 
AG, Wuppertal, West Germany ciprofloxacin and Farmitalia Carlo Erba, Milan, Italy ansamycin.

We are grateful to Dr J. Hansen for performing the stability tests with fleroxacin.

We acknowledge A. von Graevenitz for his comments on the manuscript and P. Saito for secretarial assistance.

\section{References}

Bass, J. B. (1986). American Thoracic Society: Treatment of tuberculosis and tuberculosis infection in adults and children. Ad Hoc Committee of the Scientific Assembly on Microbiology, Tuberculosis and Pulmonary Infection. American Review of Respiratory Diseases 134, 355-63.

Berlin, O. G. W., Young, L. S. \& Bruckner D. A. (1987). In-vitro activity of six fluorinated quinolones against Mycobacterium tuberculosis. Journal of Antimicrobial Chemotherapy 19, $611-5$.

Chin, N. X., Brittain, D. C. \& Neu, H. C. (1986), In-vitro activity of Ro 23-6240, a new fluorinated 4-quinolone. Antimicrobial Agents and Chemotherapy 29, 675-80.

Collins, C. H. \& Uttley, A. H. C. (1985). In-vitro susceptibility of mycobacteria to ciprofloxacin. Journal of Antimicrobial Chemotherapy 16, 575-80.

Costello, H. D., Caras, G. J. \& Snider, D. E. (1980). Drug resistance among previously treated tuberculosis patients. A brief report. American Review of Respiratory Diseases 121, 313-6.

Cynamon, M. H. (1985). Comparative in vitro activities of MDL 473, rifampin and ansamycin against Mycobacterium intracellulare. Antimicrobial Agents and Chemotherapy 28, 440-1.

Davies, S., Sparham, P. D. \& Spencer, R. C. (1987). Comparative in-vitro activity of five fluoroquinolones against mycobacteria. Journal of Antimicrobial Chemotherapy 19, 605-9.

Fenlon, C. H. \& Cynamon, M. H. (1986). Comparative in vitro activities of ciprofloxacin and other 4-quinolones against Mycobacterium tuberculosis and Mycobacterium intracellulare. Antimicrobial Agents and Chemotherapy 29, 386-8.

Gay, J. D., De Young, D. R. \& Roberts, G. D. (1984). In vitro activities of norfloxacin and ciprofloxacin against Mycobacterium tuberculosis, $M$. avium complex, $M$. chelonei, M. fortuitum and M. kansasii. Antimicrobial Agents and Chemotherapy 26, 94-6.

Goldman, K. P. (1987). AIDS and tuberculosis. British Medical Journal 295, 511-2.

Greene, J. B., Sidhu, G. S., Lewin, S., Levine, J. F., Mazur, H., Simerkoff, M. S., et al. (1982). Mycobacterium avium-intracellulare: a cause of disseminating life-threatening infection in homosexuals and drug abusers. Annals of Internal Medicine 97, 539-46.

Heifets, L. B. \& Iseman, M. D. (1985). Determination of in-vitro susceptibility of mycobacteria to ansamycin. American Review of Respiratory Diseases 132, 710-1.

Horsburgh, Ch. R., Cohn, D. L., Roberts, R. B., Masur, H., Miller, R. A., Tsang, A. Y., et al. (1986). Mycobacterium avium-M. intracellulare isolates from patients with or without acquired immunodeficiency syndrome. Antimicrobial Agents and Chemotherapy 30, 955-7.

Jones, J. A., Berry, R. V., Scott, K., Swift, M., MacCormack, J. N., Baxley, R. C., et al. (1987). Multi-drug-resistant tuberculosis-North Carolina. Morbidity and Mortality Weekly Report 35, 785-7.

Kiehn, T. E., Edwards, F. F., Brannon, P., Tsang, A. Y., Maio, M., Gold, J. W. M., et al., (1985). Infections caused by Mycobacterium avium complex in immunocompromised patients: diagnosis by blood culture and fecal examination, antimicrobial susceptibility tests, and morphological and seroagglutination characteristics. Journal of Clinical Microbiology 21, 168-73.

Masur, H., Tuazon, C., Gill, V., Grimes, G., Baird, B., Fauci, A. S., et al. (1987). Effect of combined clofazimine and ansamycin therapy on Mycobacterium avium-Mycobacterium intracellulare bacteremia in patients with AIDS. Journal of Infectious Diseases 155, 127-9.

McClatchy, J. K. (1986). Antimycobacterial drugs: mechanisms of action, drug resistance, susceptibility testing and assay of activity in biological fluids. In Antibiotics in Laboratory Medicine, 2nd edn (Lorian, V., Ed.), pp. 181-222. Williams \& Wilkins, Baltimore.

Sommers, H. M. \& Good, R. C. (1985). Mycobacterium. In Manual of Clinical Microbiology, 4th edn (Lennette E., Balows, A., Hausler, W. J. \& Shadomy, H. J., Eds), pp. 216-48. American Society for Microbiology, Washington, DC. 
Tsukamura, M. (1983). In-vitro antimycobacterial activity of a new antibacterial substance DL8280 -differentiation between some species of mycobacteria and related organisms by the DL-8280 susceptibility test. Microbiology and Immunology 27, 1129-32.

Vestal, A. L. (1981). Procedures for the isolation and identification of mycobacteria. US Public Health Service Publication. 81-8230, Center for Disease Control, Atlanta, USA.

Weidekamm, E., Portmann, R., Suter, K., Partos, C., Dell, D. \& Lücker, P. W. (1987). Singleand multiple-dose pharmacokinetics of fleroxacin, a trifluorinated quinolone, in humans. Antimicrobial Agents and Chemotherapy 31, 1909-14.

Whimbey, E., Kiehn, T. E. \& Armstrong, D. (1986). Disseminated Mycobacterium aviumintracellulare disease: diagnosis and therapy. In Current Clinical Topics in Infectious Diseases Vol. 7 (Remington, J. \& Swartz, M., Eds), pp. 112-33. McGraw-Hill, New York.

Wise, R., Lister, D., McNulty, C. A. A., Griggs, D. \& Andrews, J. M. (1986). The comparative pharmacokinetics of five quinolones. Journal of Antimicrobial Chemotherapy 18, Suppl. D, $71-81$.

Wise, R., Kirkpatrick, B., Ashby, J. \& Griggs, D. J. (1987). Pharmacokinetics and tissue penetration of Ro 23-6240, a new trifluoroquinolone. Antimicrobial Agents and Chemotherapy 31, 161-3.

Yajiko, D. M., Nassos, P. S. \& Hadley, W. K. (1987). Therapeutic implications of inhibition versus killing of Mycobacterium avium complex by antimicrobial agents. Antimicrobial Agents and Chemotherapy 31, 117-20. 